\title{
Sensitive biosensor array using surface plasmon resonance on metallic nanoslits
}

\author{
Kuang-Li Lee \\ Academia Sinica \\ Research Center for Applied Sciences \\ 128, Section 2, Academia Road \\ Nankang, Taipei 11529, Taiwan \\ and \\ National Taiwan University \\ Graduate Institute of Electro-Optical Engineering \\ Department of Electrical Engineering \\ Taipei, Taiwan
}

\section{Chia-Wei Lee}

Academia Sinica

Research Center for Applied Sciences

128, Section 2, Academia Road

Nankang, Taipei 11529, Taiwan

\section{Way-Seen Wang}

National Taiwan University

Graduate Institute of Electro-Optical Engineering

Department of Electrical Engineering

Taipei, Taiwan

\section{Pei-Kuen Wei}

Academia Sinica

Research Center for Applied Sciences

128, Section 2, Academia Road

Nankang, Taipei 11529, Taiwan

and

National Taiwan Ocean University

Department of Optoelectronics

Keelung, Taiwan

\begin{abstract}
Chip-based biosensor arrays for label-free and highthroughput detection were fabricated and tested. The sensor array was composed of a 150-nm-thick, 50-nm-gap, and 600-nm-period gold nanoslits. Each array size was $100 \mu \mathrm{m} \times 100 \mu \mathrm{m}$. A transversemagnetic polarized wave in these metallic nanostructures generated resonant surface plasmons at a wavelength of about $800 \mathrm{~nm}$ in a water environment. Using the resonant wavelength shift in the nanoslit array, we achieved detection sensitivity up to $668 \mathrm{~nm}$ per refractive index unit, about 1.7 times larger than that reported on an array of nanoholes. An antigen-antibody interaction experiment in an aqueous environment verified the sensitivity in a surface binding event. () 2007 Society of Photo-Optical Instrumentation Engineers. [DOI: $10.1117 / 1.2772296]$
\end{abstract}

Keywords: sensors; biomedical optics; detector arrays.

Paper 06345R received Nov. 24, 2006; revised manuscript received Mar. 15, 2007; accepted for publication Mar. 15, 2007; published online Aug. 31, 2007.

\section{Introduction}

Optical excitation of a surface plasmon (SP) on a thin metallic surface is widely applied for sensitive biosensing. ${ }^{1}$ The standard approach employs attenuated total reflection (ATR) in a glass prism to excite an SPW on a thin gold film coated on the prism. ATR biosensors are known to be very sensitive to surface environmental changes. The change of surface refractive index unit (RIU) can be measured on the order of $10^{-6}$ for a precise angular measurement $\left(1 \times 10^{-4}\right.$ degree $)$ or $2 \times 10^{-5}$ for a $0.02-n m$ wavelength shift in the optical spectrum. ${ }^{2}$ However, the ATR setup is typically bulky and expensive and requires a large amount of sample solution. Due to its optical configuration, it is hard to be applied for high-throughput and chip-based detections, such as DNA and protein microarrays. ${ }^{3,4}$

The studies on modern nano-plasmonics show that SPs can also be efficiently excited in metallic nanostructures. ${ }^{5-7}$ Moreover, the resonance of SPs in a periodic nanostructure causes an extraordinary transmission in certain wavelengths. The

Address all correspondence to Pei-Kuen Wei, Research Center for Applied Sciences, Academia Sinica, Taipei, 11529, Taiwan; Tel: (886) 2-27898000; E-mail: pkwei@gate.sinica.edu.tw resonant condition is decided by the nanometer aperture, period of the structure, and surface condition. Since the resonant wavelength is changed under different surface conditions, the metallic nanostructures are expected to be good sensing elements for high-throughput detections. Recently, Brolo et al. proved this concept by using an array of nanoholes in a 200nm-thick gold film. ${ }^{8}$ A sensitivity of $400 \mathrm{~nm} / \mathrm{RIU}$ was achieved by measuring the resonant wavelength shift. Light is usually cut off in the nanoholes. The enhanced transmission is attributed to the surface plasmon resonance (SPR) on the top and bottom of the metallic film. The resonance occurs when the incident wavelength and period of the nanostructure satisfy the phase-matching condition. However, in a glass biochip, the refractive indices of the substrate and outside air or water environment have different phase-matching conditions. This results in a low transmission of light and a lower SPR sensitivity for such nanohole structures.

Recently, we found that a transverse-magnetic (TM) polarized wave can directly transmit through a nanoslit without cutoff. ${ }^{9}$ Different from nanoholes, the extraordinary transmission of a TM wave was explained by the SP resonance in the

1083-3668/2007/12(4)/044023/5/\$25.00 @ 2007 SPIE 

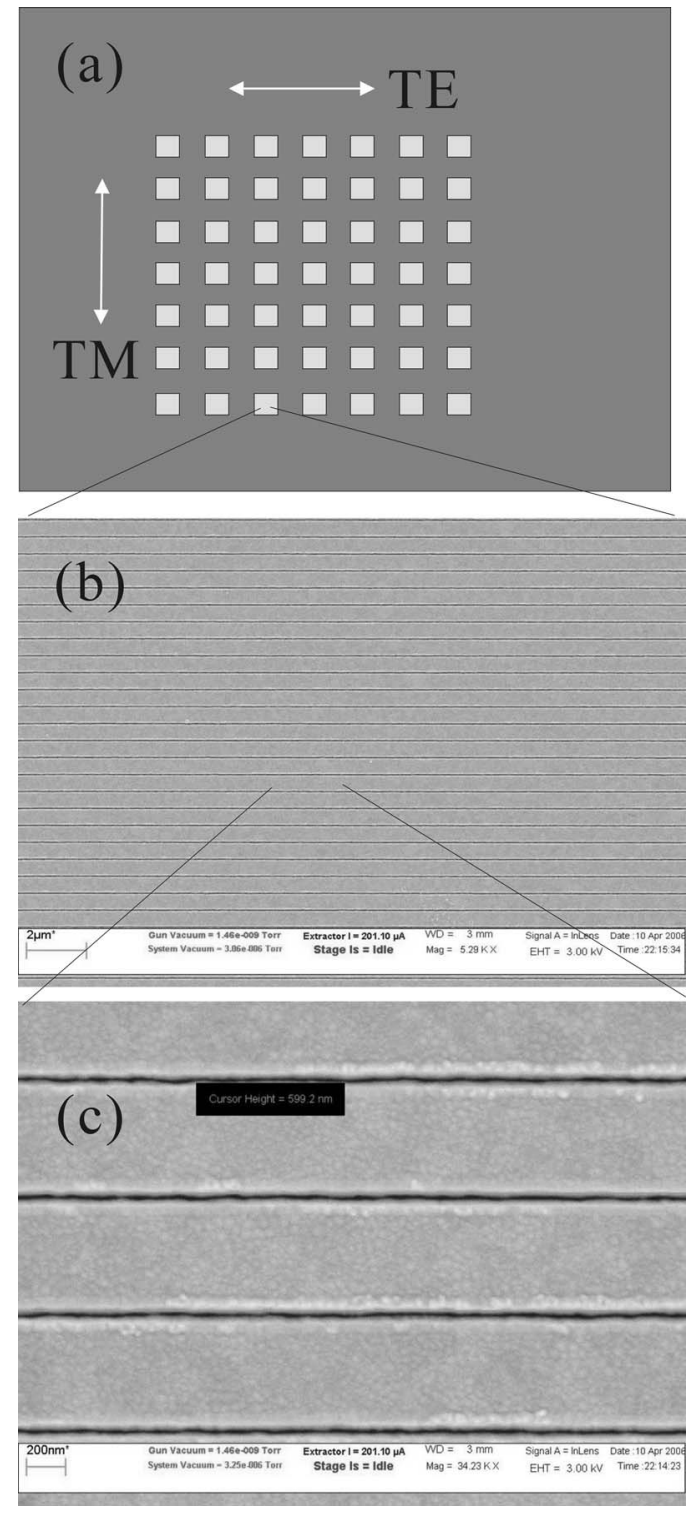

Fig. 1 (a) $7 \times 7$ nanoslit arrays on a glass substrate. The area of a nanoslit array is $100 \mu \mathrm{m} \times 100 \mu \mathrm{m}$. The separation between arrays is $100 \mu \mathrm{m}$. The TM and TE indicate the direction of incident polarization. (b) and (c) The SEM images of a nanoslit array. The period of the array is $600 \mathrm{~nm}$, and the air gap is $\sim 50 \mathrm{~nm}$.

nanoslit. The SP can also propagate along the exit metallic surface and cause interference pattern when nanoslits are close enough. ${ }^{10,11}$ If the period of the nanoslit array satisfies the phase-matching condition, constructive interference will result in an additional SPR peak in the transmission spectrum. Such SPR is only related to the outside surface condition and is decoupled from the substrate SPR mode. Thus, it is expected to have a higher sensitivity than nanohole structures. Besides, light is not cut off in the nanoslit. The transmission intensity is much stronger than that of the nanoholes. As a result, the nanoslit array is a better candidate than the nanohole array in the chip-based biosensor. In this paper, we verify this result by fabricating an SPR microarray consisting of periodic nanoslits. For a nanoslit array with a 50-nm-gap, $150-\mathrm{nm}$ thickness, and 600-nm period on a glass substrate, the

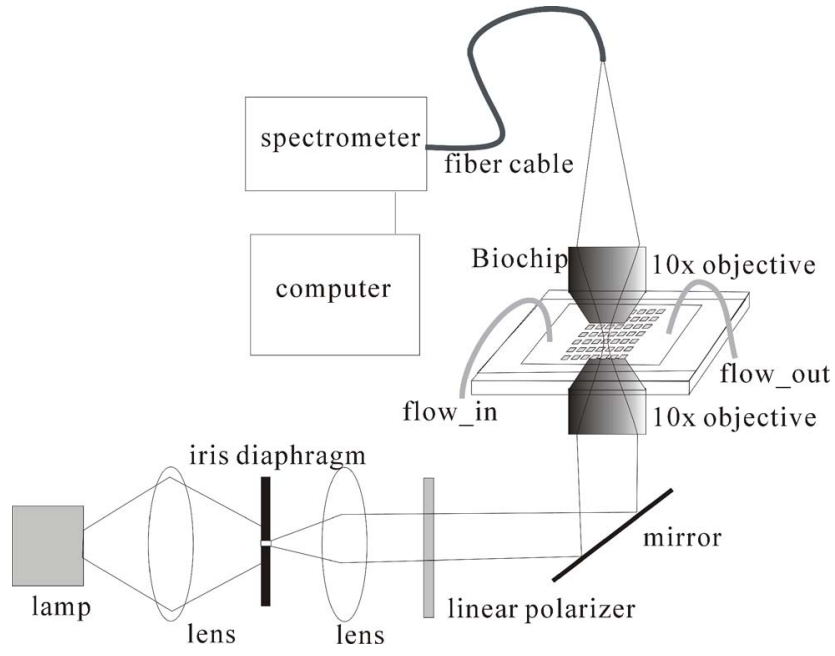

Fig. 2 The optical setup for transmission spectrum measurement. The light source is a 12-W halogen map. The iris diaphragm and 10X objective lens made an optical spot smaller than $100 \mu \mathrm{m}$ in diameter on the sample surface. The transmission light is delivered by a fiber cable and measured by a linear CCD array spectrometer.

measured sensitivity is $\sim 668 \mathrm{~nm} / \mathrm{RIU}$, about 1.7 times larger than that for the nanohole array. The measured transmission in the nanoslit array is about one order of magnitude larger than that in the nanohole array.

\section{Fabrication of Nanoslit Array}

Metallic nanoslits were fabricated by using electron beam lithography and a reactive ion etching method. We used a sodalime glass as the substrate. Because gold has poor adhesion to the glass surface, a 5-nm-thick Ti film and a 150-nm-thick gold film were sequentially deposited on the glass sample by using an electron gun evaporator. After the deposition, a 350nm-thick PMMA resist (MicroChem Corp., Newton, MA) was spin-coated. A field emission scanning electron microscope (LEO-1530) modified with a nano-pattern generating system (NPGS) was used to write nanoslits on the PMMA resist. The patterns were then transferred into $\mathrm{Ti} / \mathrm{Au}$ film by using argon sputtering in a reactive ion etching machine (Oxford Instrument, plasmalab 80plus). The power of the radiofrequency $(\mathrm{RF})$ electromagnetic wave in the reaction chamber was $200 \mathrm{~W}$. The chamber pressure was $1 \times 10^{-2}$ torr, and the flow rate of Ar gas was $40 \mathrm{sccm}$. The PMMA resist was removed by rinsing the sample in acetone for a few hours. To make sure that no PMMA resist residue remained on the gold nanoslits, we further cleaned the sample by using ozone sputtering in the RIE chamber. The sample was then put in ultrapure water, placed in an ultrasound bath for $20 \mathrm{~min}$, and purged dry by nitrogen. Figure 1 shows $7 \times 7$ slit arrays on the glass substrate. The scanning electron microscope (SEM) images show that each array consists of 600-nm-period and 50-nm-gap nanoslits. The area of the nanoslit array is $100 \mu \mathrm{m} \times 100 \mu \mathrm{m}$. The separation between adjacent arrays is $100 \mu \mathrm{m}$. This dimension is comparable with the spot size and separation in a DNA microarray. In addition to the fabrication of slit arrays, an array of nanoholes with a 600-nm 

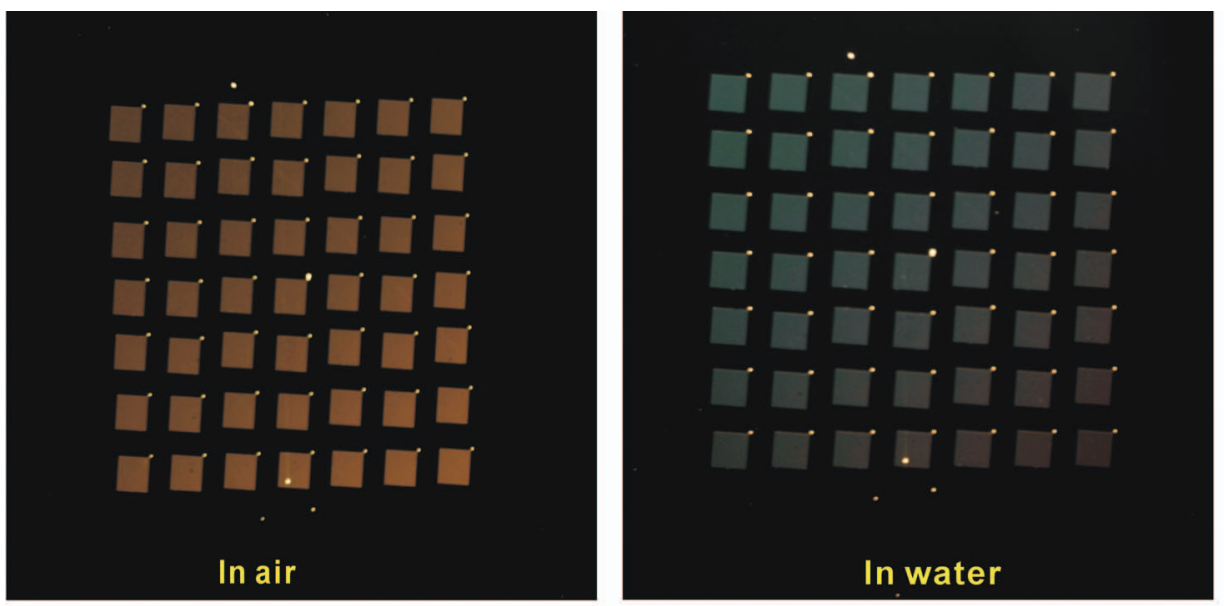

(a)

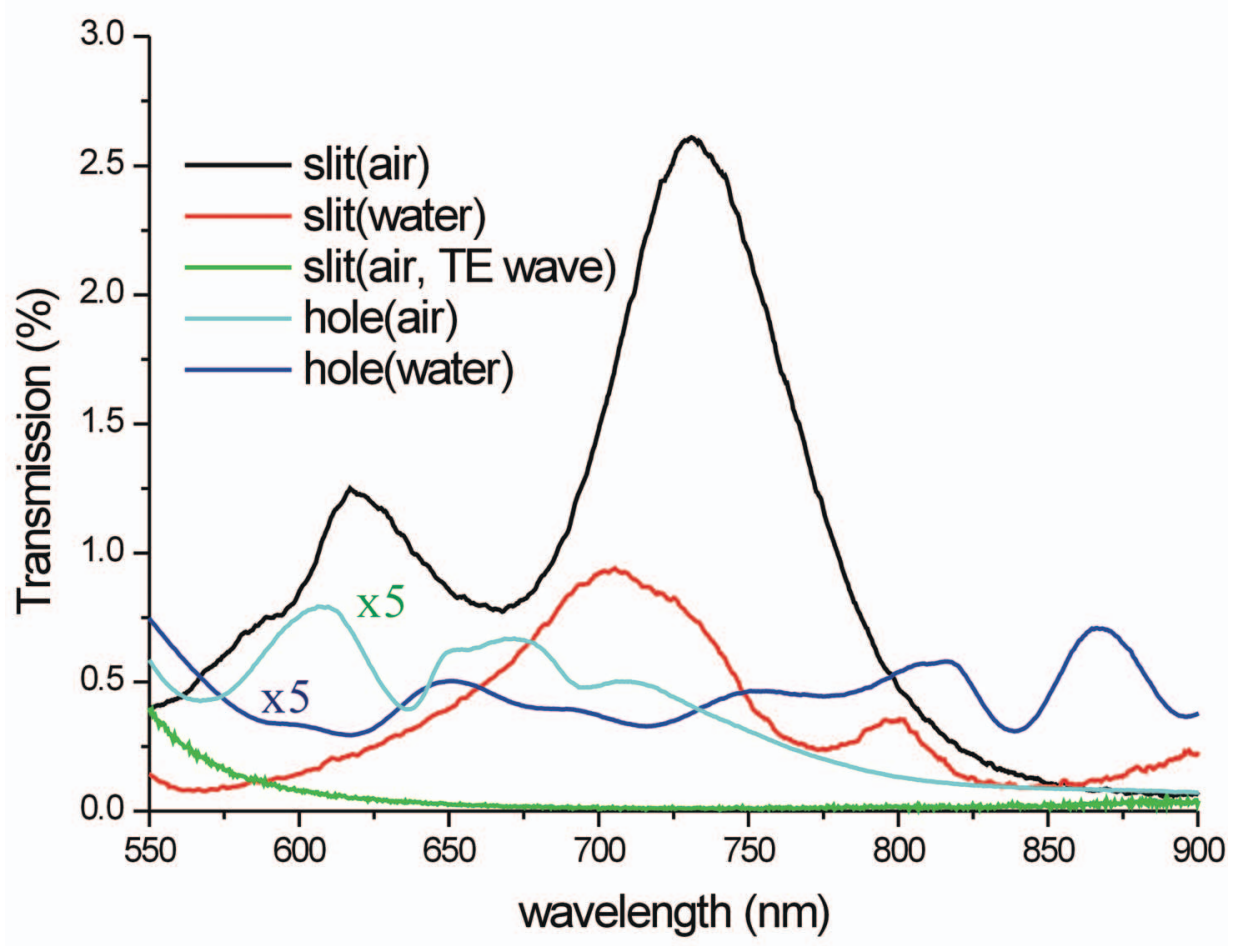

(b)

Fig. 3 (a) The transmission optical images of the $7 \times 7$ nanoslit arrays in air and water. The color difference is due to different SPR conditions on the metallic surface. (b) The measured transmission spectra of nanoslit and nanohole arrays in air and water. The green line was measured in air and TE polarized condition. The other curves were measured by TM polarized waves. The peak wavelengths show the positions for SPR on a metallic surface and cavity mode in nanoslits.

period and 100-nm-hole diameter was also made to compare their optical transmissions.

\section{Measurement Results}

The transmission spectrum for a single array was tested by using a white light source ( $12 \mathrm{~W}$, halogen lamp). Figure 2 shows the optical measurement setup. The light was spatially filtered by using a lens, an iris diaphragm, and a collimation lens. The incident polarization was controlled by a linear po- larizer. The polarized light was then focused on a single array by using a $10 \times$ objective lens. Note that to avoid the influence from other arrays, the beam size focused on the array needs to be smaller than $100 \mu \mathrm{m}$. This was accomplished by adjusting the aperture size of the diaphragm. The transmission light was then collected by another $10 \times$ objective lens and focused on a fiber cable. The transmission spectrum was taken by using a fiber-coupled linear CCD array spectrometer (BWTEK, BTC112E). 
Figure 3(a) shows the transmission optical images of $7 \times 7$ slit arrays in air and in water. It is seen that both colors are quite different due to different SPR conditions on the metallic surface. Figure 3(b) shows the measured transmission spectra of a nanoslit array in air and water under transverseelectric (TE) or TM polarization. For a TE polarized wave, the transmission is very weak because the optical wave is under a cutoff condition. The intensity drops quickly when the incident wavelength increases. For TM polarized wave in air, there are two transmission resonant peaks, at $630-\mathrm{nm}$ and 730-nm wavelength, respectively. These two modes are attributed to two different resonant conditions in the nanoslit array: the SPR on the metallic surface and the resonant cavity mode in nanoslits. ${ }^{12}$ The SPR mode is relative to the period of slit and surface condition. It occurs when incident wavelength meets the phase-matching condition; ${ }^{5}$

$$
\lambda=P \sqrt{\frac{\varepsilon_{s} \varepsilon_{m}}{\left(\varepsilon_{s}+\varepsilon_{m}\right)}},
$$

where $\lambda$ is the resonant wavelength, $P$ is the period $(=600 \mathrm{~nm})$, and $\varepsilon_{s}$ and $\varepsilon_{m}$ are the dielectric constants for the surface medium and the metal, respectively. In air, the SPR peak is at $\sim 632 \mathrm{~nm}\left(\varepsilon_{s}=1, \varepsilon_{m}=-10\right.$ for gold at the $600-\mathrm{nm}$ wavelength). Hence, the measured $630-\mathrm{nm}$ peak wavelength came from the resonant SP on the metallic surface, and the 730 -nm wavelength was the cavity mode in nanoslits. In water $\left[\varepsilon_{s}=(1.32)^{2}\right.$ and $\varepsilon_{m}=-25$ for gold at the 800 -nm wavelength], the estimated SPR peak was at $\sim 821 \mathrm{~nm}$. The measured $807-\mathrm{nm}$ peak wavelength meets quite well with the prediction of the SPR mode. The figure also shows the measured results for a 600-nm-period nanohole array. It can be seen that an SPR peak also appears near the $600-\mathrm{nm}$ wavelength in air. In water, there is an SPR peak near $820 \mathrm{~nm}$. But the transmission intensity is about one order of magnitude smaller than that in the nanoslit array.

In most SPR biosensors, an aqueous environment is preferred. Hence, the sensitivity at the 807-nm-wavelength SPR mode was tested. Figure 4(a) shows the transmission spectra in water and different methanol/water mixtures for TM polarized waves. There was an apparent redshift when the outside medium changed from water to a methanol-rich medium. Figure 4(b) shows the peak wavelengths against the refractive index of the outside medium. The slope of the fitting curve shows that the RIU sensitivity is $668.9 \mathrm{~nm} /$ RIU. This value is $\sim 1.7$ times larger than the array of nanoholes, which is $400 \mathrm{~nm} / \mathrm{RIU}^{8}{ }^{8}$ The red shift and sensitivity can be predicted well by Eq. (1). In most cases, the absolute value of $\varepsilon_{m}$ is much larger than $\varepsilon_{s}$. Hence, the change to the SPR wavelength $(\Delta \lambda)$ can be approximated by

$$
\Delta \lambda=\lambda_{1}-\lambda_{2} \sim P\left(\sqrt{\varepsilon_{s_{1}}}-\sqrt{\varepsilon_{s 2}}\right)=P \Delta n,
$$

where $\Delta n$ is the change of the refractive index. The predicted sensitivity is $\sim 600 \mathrm{~nm} / \mathrm{RIU}$ for the 600 -nm-period nanoslit array. This value is close to the measured result and is comparable with grating coupled-based SPR sensors. ${ }^{2}$ It is noticeable that Eq. (2) indicates that the SPR sensitivity is proportional to the period. An increase in the period can increase the sensitivity. However, if the period is larger than $800 \mathrm{~nm}$, the SPR wavelength in water will be in the infrared region. A

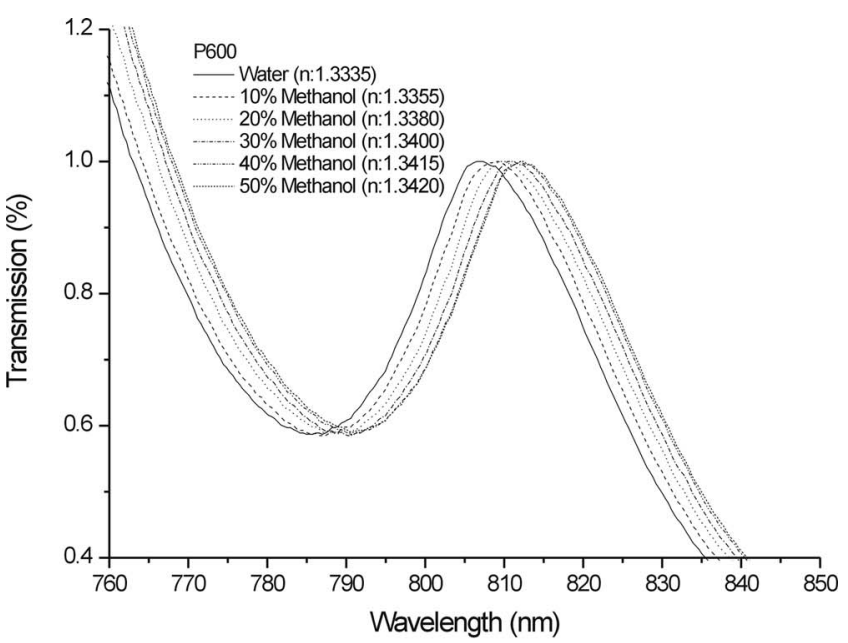

(a)

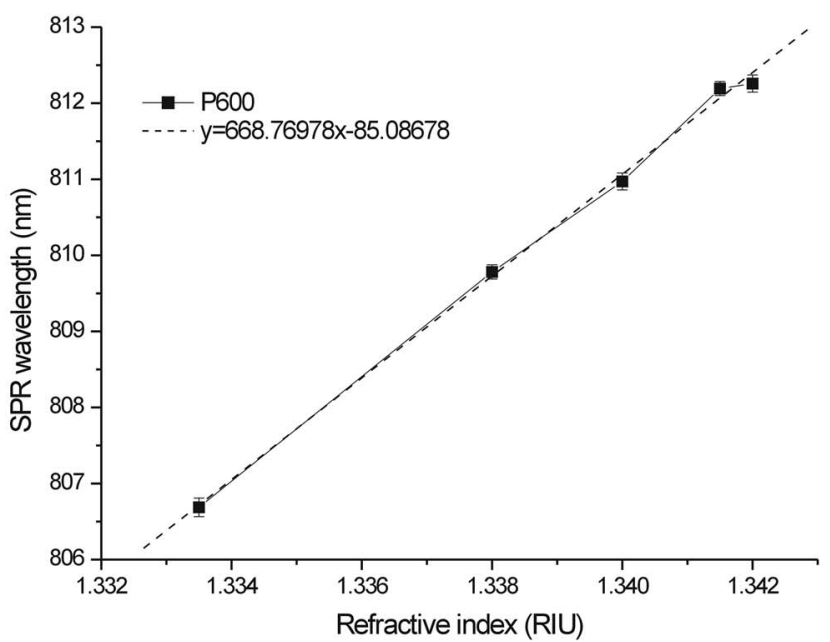

(b)

Fig. 4 (a) The transmission spectra in water and different methanol/ water mixtures for TM polarized wave. There was an apparent red shift when the outside medium was changed from water to a methanol-rich medium. (b) The peak wavelengths against the refractive index of the outside medium. The slope of the fitting curve shows that the RIU sensitivity is $688.9 \mathrm{~nm} /$ RIU.

sensitive and high-resolution IR spectrometer is necessary for the measurement. In our experiments, the period was $600 \mathrm{~nm}$, and the SPR wavelength in water was $\sim 820 \mathrm{~nm}$. The commercial CCD spectrometer is sensitive to this wavelength.

It is worthwhile to compare the nanoslit-based sensor with the grating coupled-based sensor. For a grating coupled device, the phase-matching condition is written as ${ }^{13}$

$$
\sin \theta+\frac{\lambda_{0}}{\Lambda}=\sqrt{\frac{\varepsilon_{s} \varepsilon_{m}}{\left(\varepsilon_{s}+\varepsilon_{m}\right)}},
$$

where $\theta$ is the incident angle and $\Lambda$ is the period of the grating. Hence, a precise control of the incident angle is necessary to excite the surface plasmon. In the nanoslit device, the SPR wavelength is insensitive to the incident angle. In addition, the spectrum in a grating coupled device is measured by the reflection light from the top surface. Scattering of the aqueous medium would cause substantial noise in the measurement. 


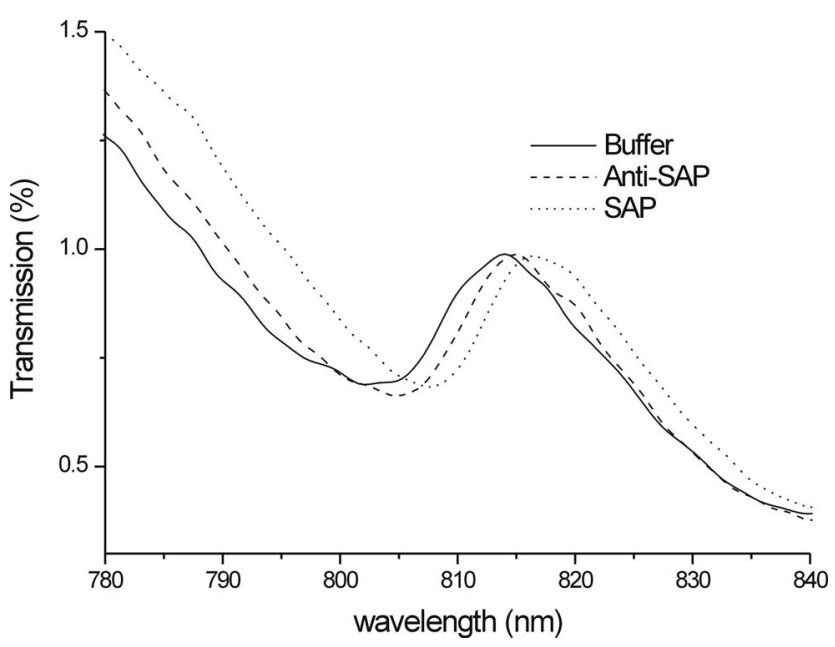

Fig. 5 The measured spectra for different surface conditions on a gold nanoslit array. The anti-SAP on the gold surface made an $\sim 1-\mathrm{nm}$ red shift. The shift was increased to $2.4 \mathrm{~nm}$ when the SAP was bound to anti-SAP. All the measurements were taken in the same buffer solutions.

On the contrary, the spectrum in nanoslit device is taken in the transmission mode. Transmission light is dominant by the evanescent surface plasmonic waves. The scattering of the aqueous medium has a small effect on this device. The major drawback of the nanoslit-based sensor is the fabrication cost. Most nanostructures are made by using electron beam lithography or the focused ion beam method. For the grating coupling device, the grating is easily fabricated by using the two laser beam interference method.

The aforementioned sensitivity was obtained by fulfilling the fluidic channel with different refractive index media. For verifying the surface sensitivity, an antigen-antibody interaction was tested. The antigen was serum amyloid $\mathrm{P}$ component (SAP), a $25-\mathrm{kDa}$ pentameric protein. It was first identified as the pentagonal constituent of in vivo pathological deposits ${ }^{14}$ and is thought to be an important contributor to the pathogenesis of a related group of diseases called the amyloidoses. ${ }^{15}$ We applied the nanoslit array to detect the anti-SAP and SAP interactions on the gold surface. In the experiment, pure water and buffer solution, $10 \mathrm{mM} \mathrm{HK} \mathrm{PO}_{4}$, were first injected to the fluidic channel to clean the gold surface. The transmission spectrum of the nanoslit array in the buffer solution was taken. Then $0.125 \mu \mathrm{M}$ of anti-SAP in the buffer solution was injected into the channel. The sample was placed overnight in order to make anti-SAP bind well to the gold surface. A new buffer without anti-SAP was then injected two times to remove the unbound antibodies. The spectrum of anti-SAP on gold film in the buffer solution was taken. Next, $2.09 \mu \mathrm{M}$ SAP was injected into the channel. After several hours of displacement for the antigen-antibody interactions, the unbound SAP was washed away by twice filling the channel with new buffer. The transmission spectrum was then taken. Figure 5 shows the measured spectra for different surface con- ditions. The anti-SAP on a gold surface made an $\sim 1-\mathrm{nm}$ red shift of the SPR peak. The shift was increased to $2.4 \mathrm{~nm}$ when SAP was bound to anti-SAP. The 25-kDa-sized SAP resulted in a 1.4-nm wavelength shift. It implies that if the resolution of a spectrometer is $0.1 \mathrm{~nm}$, the gold nanoslit array can detect $\sim 2$-kDa-sized biomolecules attached to the surface.

In summary, we studied the surface sensitivity of a gold nanoslit array in an aqueous environment. The RIU test shows that the sensitivity is 1.7 times larger than that of an array of nanoholes. The anti-SAP and SAP interaction test verified good surface sensitivity for bio-affinity studies. Our measurements were taken in a small area of $100 \mu \mathrm{m} \times 100 \mu \mathrm{m}$, and the acquisition time was shorter than $1 \mathrm{~s}$. The high-throughput detection is thus feasible when thousands of arrays of nanoslits were fabricated on a glass chip.

\section{Acknowledgments}

This research is supported by the National Science Council, Taiwan (Grant no. NSC 95-2215-E-001-001) and the Thematic Project of Academia Sinica, Taiwan.

\section{References}

1. W. Knoll, "Interfaces and thin films as seen by bound electromagnetic waves," Аnnu. Rev. Phys. Chem. 49, 569-639 (1998).

2. J. Homola, S. S. Yee, and G. Gauglitz, "Surface plasmon resonance sensors: review," Sens. Actuators B 54, 3-15 (1999).

3. M. Schena, D. Shalon, R. W. Davis, and P. O. Brown, "Quantitative monitoring of gene expression patterns with a complementary DNA microarray," Science 270, 467-470 (1995).

4. M. B. Gavin and L. S. Stuart, "Printing proteins as microarrays for high-throughput function determination," Science 289, 1760-1763 (2000).

5. T. W. Ebbesen, H. J. Lezec, H. F. Ghaemi, and T. Thio, "Extraordinary optical transmission through sub-wavelength hole arrays," $\mathrm{Na}$ ture (London) 391, 667-669 (1998).

6. S. Kawata, Near-Field Optics and Surface Plasmon Polaritons (Springer, New York, 2001).

7. H. J. Lezec, A. Degiron, E. Devaux, R. A. Linke, L. Martin-Moreno, F. J. Garcia-Vidal, and T. W. Ebbesen, "Beaming light from a subwavelength aperture," Science 297, 820-822 (2002).

8. A. G. Brolo, R. Gordon, B. Leathem, and K. L. Kavanagh, "Surface plasmon sensor based on the enhanced light transmission through arrays of nanoholes in gold films," Langmuir 20, 4813-4815 (2004).

9. P. K. Wei, H. L. Chou, and W. S. Fann, "Optical near field in nano metallic slits," Opt. Express 10, 1418-1424 (2002).

10. H. F. Schouten, N. Kuzmin, G. Dubois, T. D. Visser, G. Gbur, P. F. A Alkemade, H. Blok, G. W. Hooft, D. Lenstra, and E. R. Eliel, "Plasmon-assisted two-slit transmission: Young's experiment revisited," Phys. Rev. Lett. 94, 053901 (2005).

11. K. M. Chae, H. H. Lee, S. Y. Yim, and S. H. Park, "Evolution of electromagnetic interference through nano-metallic double-slit," Opt. Express 12, 2870-2879 (2004).

12. F. Yang and J. R. Sambles, "Resonant transmission of microwave through a narrow metallic slit," Phys. Rev. Lett. 89, 063901 (2002).

13. R. G. Hunsperger, Integrated Optics: Theory and Technology, 5th ed., Springer, New York (2002)

14. E. S. Cathcart, T. Shirahama, and A. S. Cohen, "Isolation and identification of a plasma component of amyloid," Biochim. Biophys. Acta 147, 392 (1967).

15. M. B Pepys, D. R. Booth, W. L. Hutchinson, J. R Gallimore, P. M. Collins, and E. Hohenester, "Amyloid P component. A critical review," Amyloid 4, 274-295 (1997). 